Real Analysis Exchange

Vol. 23(2), 1997-1998, pp. 779-781

Henry Fast, 1745 De Marietta Ave, \# 1, San Jose CA 95126. e-mail:

hfast@inow.com

\title{
A FEW REMARKS ABOUT EQUALITY OF INDICATRICES OF TWO FUNCTIONS AND A CONJECTURE.
}

\begin{abstract}
Having defined for a couple of functions non co-supportivity in a given direction, we construct for any finite set of directions a couple $\mathrm{f}$ and $\mathrm{g}$ of possibly very regular, continuous except finite many points functions, which in any of these directions are non co-supportive and have the same indicatrix. Adding the requirement that both be continuous (on the joint interval of definition), we are still able to produce such a construction for two directions, but two seems to be the limit: it seems that such a result could not be had for three directions.
\end{abstract}

Let $f$ be a function defined over a subset of $R$. The $\theta$-directional indicatrix of $f$, where $0 \leq \theta<\pi / 2$, is defined as the function $N_{f, \theta}(y)=\operatorname{card}\{x: f(x)=$ $x \tan \theta+y\}$ taking finite integers or $\infty$ as values.

A couple of functions $f, g$ both defined over the same subset of $R$ is $\theta$-non co-supportive if the graph of at least one of the two has a support line of direction $\theta$ which supports it at a point not belonging to the other graph.

(note: this property implies that $f$ and $g$ are not identical. We are dealing in this note with the issue of the possibilities for two distinct functions to have the same directional indicatrices. The non co-supportiveness condition eliminates the trivial cases of such occurrences. )

Proposition 1. For any finite sequence of directions $0=\theta_{0}<\cdots<\theta_{n}<\pi / 2$ there is a couple of non co-supportive in any of these directions continuous functions which have the same directional indicatrices in all these directions.

The Construction. We construct in the (complex plane) $R^{2}$ a $2^{n}$-point set $W_{n}$ by the following inductive construction.

Set $W_{0}=\{0\}$ and define $W_{k}=W_{k-1} \cup\left(W_{k-1}+r_{k} e^{\theta_{k} i}\right), k=0, \ldots, n$ choosing the positive numbers $r_{k}$ such that the $W_{k-1}$ and $W_{k}+r_{k} e^{\theta_{k} i}$ and

Mathematical Reviews subject classification: 26A15

Received by the editors September 3, 1997 
also their real-axis projections become disjoint. ( $W_{n}$ is a projection to $R^{2}$ from $R^{n}$ of an $n$-dimensional parallelepiped in such a way that all its $2^{n}$ vertices project on different points which distinct real parts.)

Imagine the vertices of the $n$-dimensional parallelepiped of which $W_{n}$ is a projection colored red and white in such a way that two endpoints of an edge get different colors (this can be done in two ways, depending which color gets the first point.) after which half of the vertices become 'red', the other half 'white'. Transfer these colors by projection onto $W_{n}$ and let $W_{n}^{\prime}$ and $W^{\prime}{ }_{n}$ be its 'red' and 'white' halves.

Over each point of $W_{n}$ place an identical copy of a minuscule triangle pointed up. By construction the bases of these triangles project on the real axis as disjoint intervals. The triangles inherit the 'colors' of their respective points.

The unions of the 'red' and of the 'white' triangles form by themselves graphs of two functions $F$ and $G$.

The so created couple $F, G$ is $\theta_{k}, k=0, \ldots, n$-non co- supportive and the directional indicatrices of $F$ and $G$ are the same in all of these directions, provided that the base of the minuscule triangle used in the construction was sufficiently small.

Proposition 2. There are two functions defined over the same interval, both continuous and non co-supportive in 0 and $\theta, 0<\theta</ p i / 2$ directions, whose indicatrices in both of these directions are the same.

The Construction. Let $h$ be an arbitrary function defined over $[0,2]$ with values $h(0)=h(2)=0$

The two functions: $f_{1}(x)=h(x)+x / 2$ and $g_{1}(x)=h(2 x)-x$ are defined respectively over the $[0,2]$ and $[0,1]$ intervals. The line $l_{0}=\{y: y=2 x+t\}$ where $-3 \leq t \leq 0$ meets the graphs of both functions. Let $\left(x_{0}, g_{1}\left(x_{0}\right)\right)=$ $\left(x_{0}, h\left(2 x_{0}\right)-x_{0}\right)$ be the point at which it meets the graph of $g_{1}$. Then the parallel to it line: $l_{1}=\left\{y: y=2 x+t-h\left(2 x_{0}\right)\right\}$ meets the diagonal $\{y: y=$ $-x\}$ at the point $\left(x_{0},-x_{0}\right)$ and meets the line $\{y: y=(1 / 2) x\}$ at the point $\left(2 x_{0}, x_{0}\right)$.

But then $l_{0}$ carries the point $\left(2 x_{0}, h\left(2 x_{0}\right)+x_{0}\right)=\left(2 x_{0}, f_{1}\left(2 x_{0}\right)\right)$ of the graph of $f_{1}$ corresponding to the point $\left(x_{0}, g_{1}\left(x_{0}\right)\right)$. And this implies that the indicatrices of $f_{1}$ and of $g_{1}(x)$ in direction $\theta=\arctan 2$ are equal.

Extend $f_{1}$ and $g_{1}$ anti-symmetrically by setting: $f_{2}(x)=-f_{1}(-x)$ for $x \in[-2,0]$ and $g_{2}(x)=-g_{1}(-x)$ for $x \in[-1,0]$. Evidently, the arctan 2directional indicatrices of the extended pair $f_{2}, g_{2}$ are equal as well.

Define four additional functions: $f_{3}(x)=f_{2}(x-3)$ for $x \in[1,3], f_{4}(x)=$ 
$f_{1}(x+3)$ for $x \in[-3,-1], g_{3}(x)=g_{2}(x-3)$ for $x \in[2,3], g_{4}(x)=g_{1}(x+3)$ for $x \in[-3,-2]$. Set now:

$$
\Phi(x)= \begin{cases}f_{4}(x) & \text { for } x \in[-3,-1] \\ g_{2}(x) & \text { for } x \in[-1,0] \\ f_{1}(x) & \text { for } x \in[0,2] \\ g_{3}(x) & \text { for } x \in[2,3]\end{cases}
$$

and

$$
\Psi(x)= \begin{cases}g_{4}(x) & \text { for } x \in[-3,-2] \\ f_{2}(x) & \text { for } x \in[-2,0] \\ g_{1}(x) & \text { for } x \in[0,1] \\ f_{3}(x) & \text { for } x \in[1,3] .\end{cases}
$$

$\Phi$ and $\Psi$ are defined over the interval $[-3,3]$ functions. The 0-directional and $\arctan 2$-directional indicatrices of both are identical. They are continuous if the original $f_{1}$ and $g_{1}$ are, but one may have them as rough as one wishes The two functions may be made non co-supportive in any direction one may wish them to be.

Note: Clearly, the choice of arctan 2 as the direction was dictated only by the need to simplify the exposition. For any other direction the same may be obtained by subjecting the above construction to an appropriate horizontal affine sheet.

Conjecture 1. The two directions the Proposition 2 go as far as possible; a similar result can not be obtained for three directions. (But to prove it seems to me to be far from trivial.) 